\title{
Assessment of Health of Friction Pair in Sliding Bearing Using Vibration Sensor and Continuous Wavelet Transform Time-frequency Images
}

\author{
Chaoming Huang, ${ }^{1}$ Yongtao Liao, ${ }^{2}$ Qingtao Li, ${ }^{1}$ \\ Hongliang $\mathrm{Yu}^{3}{ }^{3 *}$ Jianbin Liao, ${ }^{3}$ and Chih-Cheng Chen ${ }^{4,5^{* *}}$ \\ ${ }^{1}$ College of Marine Engineering, Dalian Maritime University, Dalian Liaoning 116026, China \\ ${ }^{2}$ CCCC Shanghai Dredging Equipment Industry Co., Ltd., Shanghai 200137, China \\ ${ }^{3}$ College of Marine Engineering, Jimei University, Fujian 361021, China \\ ${ }^{4}$ Department of Aeronautical Engineering of Chaoyang University of Technology, Taichung 413310, Taiwan \\ ${ }^{5}$ School of Information Engineering, Jimei University, Xiamen, Fujian 361021, China
}

(Received April 29, 2020; accepted August 18, 2020)

Keywords: frictional vibration, feature extraction, time-frequency image, image segmentation, sliding bearing

A new method was proposed to find the characteristic parameters of the vibration induced by the friction and wear of a sliding bearing. The parameters were obtained from the data of vibration signals using vibration sensors and processed with the continuous wavelet transform (CWT) and image technology. We collected the vibration signal of a ZCHSnSb8-8 bearing alloy and drew three-dimensional images with the time-frequency spectrum. Then, the important features were extracted by image segmentation technology. The parameters corresponding to the features were calculated with an algorithm to find the relationship between the parameters and the vibration characteristics. The results in this study revealed that the parameters of the vibration signal quantitatively represented the vibration characteristics of the sliding bearing caused by friction and wear. The new method is useful for assessing the health of a sliding bearing that has been deteriorated by the friction and wear of its friction pair.

\section{Introduction}

Sliding bearings are used to move two surfaces relative to each other without rolling contact. They are widely applied to machines that have rotating shafts. ${ }^{(1)}$ The vibration of a sliding bearing is unavoidable because of the wear of the friction pair in the bearing. The wear causes common faults that affect the performance and operation of machines. The conventional method of monitoring the extent of the wear is to analyze the surface morphology of the friction pair or analyze the lubricating oil. The changes in surface morphology and oil properties help verify the extent of the wear of the friction pair. The surface morphology of the friction pair is analyzed by electronic microscopy, scanning electron microscopy (SEM), and/or X-ray spectroscopy. In this method, microstructures on the surface are observed and analyzed to assess the extent of the wear of the friction pair. However, it is necessary to disassemble the

\footnotetext{
*Corresponding author: e-mail: yu1202@hotmail.com

${ }^{* *}$ Corresponding author: e-mail: ncue.anko@gmail.com https://doi.org/10.18494/SAM.2020.2936
} 
pair to observe the morphology. The lubricating oil is analyzed to investigate the physical and chemical properties of wear particles. The size and shape of the particles are analyzed to assess the extent of wear. However, the lubricating oil must be sampled and sent to a lab for microscopic analysis. To obtain the sample, the sliding bearing must be disassembled, which may affect the performance of the machine. Moreover, the result of the analysis varies on how and where the oil is sampled.

Thus, a new method is required to monitor and diagnose the health of a sliding bearing by real-time analysis. Many researchers have attempted to develop such a method using vibration sensors. The vibrations of sliding bearings are caused by imbalance, misalignment, wear, and looseness that are generated by repeatedly rotating or reciprocating movements. Vibration sensors detect the frequency, amplitude, and impact of the vibrations on the key components of machines such as motors, bearings, and gears. These parameters provide valuable information to diagnose faults and monitor the condition of sliding bearings. To obtain the parameters, the data from the sensors are processed to extract and study information on the wear. This process needs the laws of vibrations to obtain the intrinsic relationship between the data and the parameters. ${ }^{(2)}$

Researchers have proposed various methods based on the power spectrum, ${ }^{(3)}$ empirical mode decomposition (EMD), ${ }^{(4)}$ and single fractal and multifractal spectra ${ }^{(5,6)}$ to obtain information on the health of machines affected by vibrations. These methods have the following limitations: (1) power spectrum analysis cannot provide the time domain information of vibration signals; (2) pattern aliasing occurs when EMD methods are used to decompose the vibration signals into several eigenmode components to obtain failure frequency components; (3) in methods based on single and multifractal spectra, the method of calculating the fractal scalefree interval affects the accuracy of the results, and the fractal algorithm is complicated and difficult to realize. ${ }^{(6-9)}$

Therefore, some researchers have proposed the use of secondary images obtained from these methods to diagnose the health of sliding bearings. In this method, statistical parameters are extracted from histograms and images, giving information on the friction and wear of the bearings with fewer computations. ${ }^{(10-14)}$ However, the secondary images cannot be directly processed to extract the parameters, and the noise of the images is not eliminated effectively.

To solve the problems of previous methods, we propose time-frequency image processing using the continuous wavelet transform (CWT) as a quantitative analysis. This method uses segmented images to eliminate noise and extract parameters efficiently. In addition, the method enables vibration signals from a group of machines to be obtained and analyzed simultaneously.

\section{Methods}

We used an MMW-1 vertical universal friction and wear tester manufactured by Jinan Liangong Testing Technology Co., Ltd. ${ }^{(15)}$ to test a sliding bearing made of ZCHSnSb8-8 tin-based alloy. We used a ring-type friction pair with a ring on the upper side and a disk on the lower side. The sliding bearing was made of a $45 \#$ steel block. ${ }^{(16)}$ The disk had a diameter of $32 \mathrm{~mm}$ and a thickness of $10 \mathrm{~mm}$. The average roughness $(R a)$ of the surface of the disk 
was $0.12 \mu \mathrm{m}$, and the surface hardness was $24 \mathrm{HV}$ according to a Vickers hardness test. The ring had the outer and inner diameters of 28 and $25 \mathrm{~mm}$, respectively, and a thickness of 5 $\mathrm{mm}$. Ra was $1.0 \mu \mathrm{m}$ and the hardness was $315 \mathrm{HV}$. The test conditions were as follows: the test speed was 300 revolutions per minute (rpm), the test load was $120 \mathrm{~N}$, the running time was 20 min, and immersion lubrication was used as the lubrication method. The torque and frictional vibrations were recorded every $2 \mathrm{~min}$. The surface morphology of the bearing after the test was measured using a metallographic microscope. Wear particles were analyzed using an FTP-X2 spectrometer and an OLYMPUS BX40 microscope. The vibration signal generated during the test was collected with INV YJ9A7024 and YJ9A7022 acceleration sensors and a Beijing Oriental INV306U vibration signal test system with a sampling frequency of $5.12 \mathrm{kHz}$.

Tests were carried out with two different lubrication methods: test A with immersion lubrication and test B with boundary lubrication using CD40 lubricating oil. A schematic diagram of the test is shown in Fig. 1.

\section{Calculation of Parameters from Vibration Signals}

\subsection{Combined sensor signals}

We installed a vibration accelerometer sensor to obtain the data of vibration signals, which were induced by friction and wear. The position and orientation of the sensor are important for obtaining information effectively. We placed the sensor as close as possible to the contact position of the ring and the disk to maximize the intensity of the vibration signals obtained from them. Vibration signals in both the tangential and normal directions were collected simultaneously. It was found that the tangential and normal vibration signals had a strong correlation. Thus, signals in these two directions can be fused by the vector synthesis method. The fused sensor signal from the two directions provided more information on the vibration than the signal from only one direction. Figures 2(a) and 2(b) show the signals from the sensors, while Fig. 2(c) shows the fused signal.

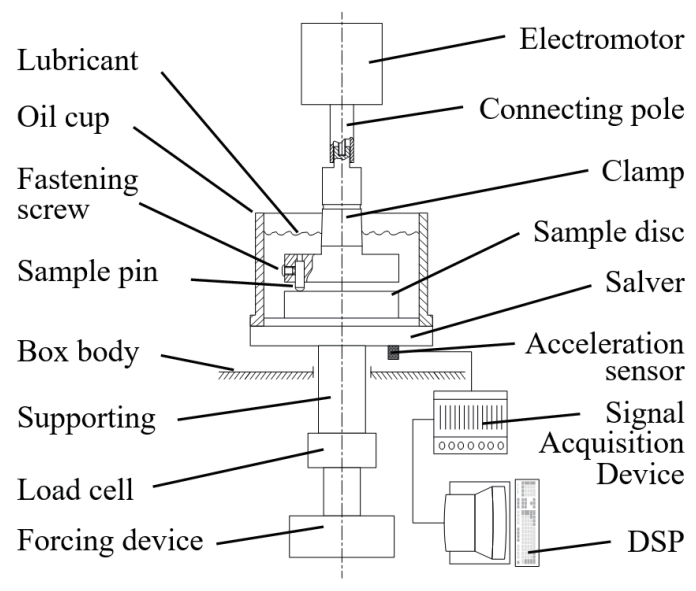

Fig. 1. Schematic diagram of test. 


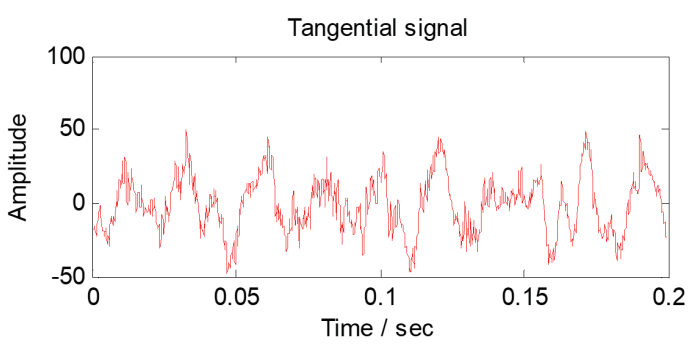

(a)

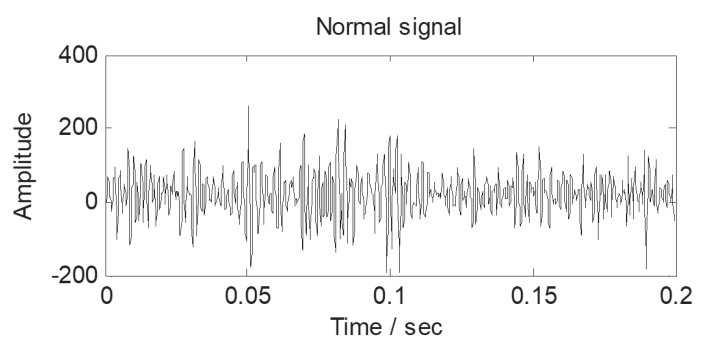

(b)

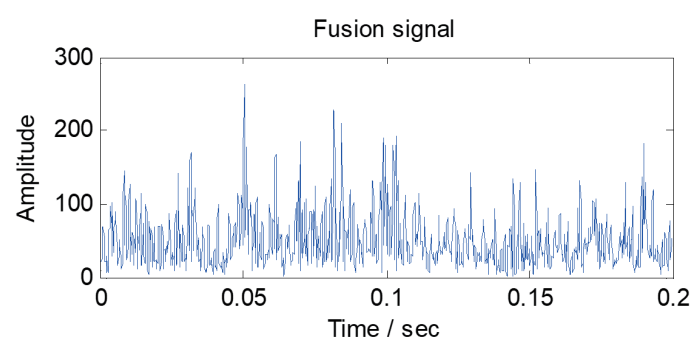

(c)

Fig. 2. (Color online) Vibration signals. (a) Tangential vibration signal. (b) Normal vibration signal. (c) Fused signal from tangential and normal vibration signals.

\subsection{CWT time-frequency images}

To obtain information on the health of the friction pair from the wear status, we used time-frequency images (TFIs) from which the parameters of the vibration were calculated. The measured signals were nonlinear and nonstationary with a wide distribution of the vibration frequency. The Morlet wavelet was adopted for the CWT of the vibration signals. ${ }^{(17)}$ The CWT is usually used to characterize frictional vibration by analyzing the signals of different frequency intervals through scale expansion and contraction. The CWT in this study is expressed as

$$
W_{\psi} f(a, b)=\left\langle f, \psi_{a, b}\right\rangle=|a|^{-1 / 2} \int_{-\infty}^{+\infty} f(t) \psi^{*}\left(\frac{t-b}{a}\right) d t, a \neq 0,
$$

where $\psi(t)$ is the parent wavelet used as the Morlet wavelet, $*$ is the conjugate operation, and $a$ is a scale factor corresponding to the frequency $f_{a}$. Here, $f_{a}$ is defined as

$$
f_{a}=\frac{f_{c} \times f_{s}}{a}
$$

where $f_{c}$ is the central frequency of the parent wavelet, $f_{s}$ is the sampling frequency, $b$ is a time shift factor that corresponds to time $t$, and $f(t)$ is the signal to be analyzed. By changing the scale factor and the time shift factor, $f(t)$ is analyzed in different frequency and time domains. 
The time-frequency spectrum is a joint function of time and frequency. It describes the vibration intensity or the energy density of vibration signals at different times and frequencies. In accordance with the above analysis, the energy spectrum of the CWT is defined as

$$
S_{\psi}\left(t, f_{a}\right)=\left|W_{\psi} f\left(f_{a}, t\right)\right|^{2} .
$$

The three-dimensional surface spectrum and its equivalent formed by the energy spectrum of the CWT are used to characterize the time-frequency property of the signal on the timefrequency phase plane. Figure 3 shows TFIs of the frictional vibration generated by the CWT method. Figures 3(a) and 3(b) correspond to the three-dimensional time-frequency spectrum and isochronal spectrum of frictional vibration signals of test A (with immersion lubrication), while (c) and (d) correspond to those of test B (with boundary lubrication), respectively. The comparison of the TFIs of the two tests with different lubrication methods revealed significant differences in energy intensity, spatial texture, and geometry.

\subsection{Characterization of vibration signals}

The above images reveal areas of different energy and frequency characteristics. These areas are some small discrete parts of an entire TFI, having meaningful information to be analyzed. Thus, we applied image segmentation based on the grayscale and geometrical distribution to the

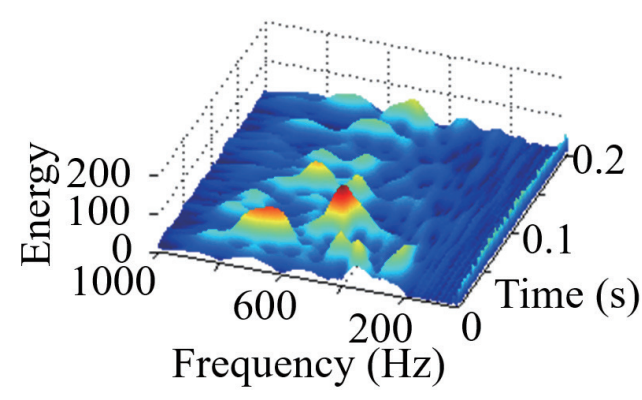

(a)

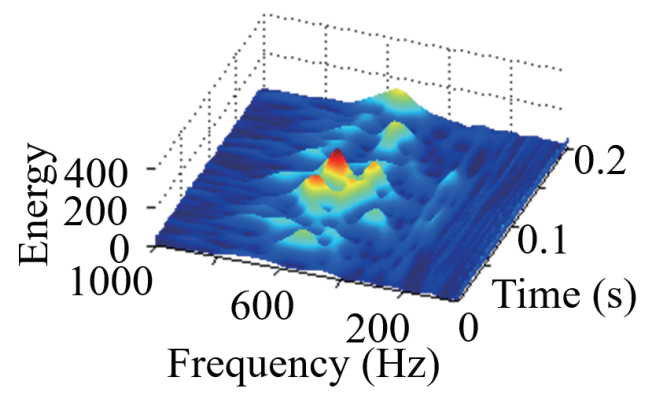

(c)

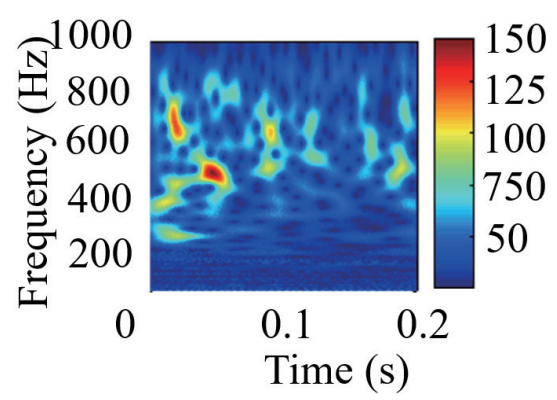

(b)

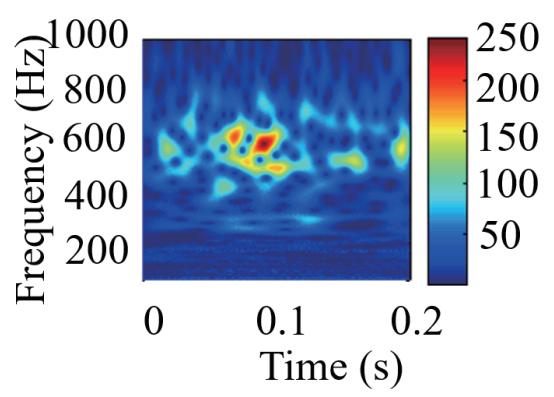

(d)

Fig. 3. (Color online) CWT TFIs of frictional vibration signals. (a) 3D surface TFI. (b) Equivalent TFI. (c) 3D surface TFI. (d) Equivalent TFI. [(a), (b) from test A and (c), (d) from test B]. 
TFIs. The segmented image was divided into several disjoint areas, with similar geometrical distribution characteristics in the same area, but significant differences among the areas. ${ }^{(18)}$ This method based on the gray-level threshold enabled the calculation to be simple with high operation efficiency. In this study, we used the Otsu threshold segmentation method for the CWT TFIs, which maximizes the variance between the areas and the background. Figure 4 shows the segmentation of the TFIs and the characteristics extracted from Fig. 3. In the figure, (a) and (b) correspond to test A, and (c) and (d) correspond to test B.

The comparison of Figs. 3 and 4 revealed that the image segmentation extracted information showing the dominance of energy in the TFIs. The background noise was suppressed and the useful information was retained. In addition, from the results of the tests on frictional vibrations shown in Figs. 4(b) and 4(d), the morphological and spatial characteristics showed significant differences in frequency and energy in the two tests. Thus, we used the results from the CWT TFIs obtained in the two tests to calculate parameters to quantitatively analyze the vibration of the sliding bearing.

\subsection{Parameters of vibration signals}

Using the CWT with the image segmentation method, we obtained some segmented objects with shape and spatial distribution characteristics of the vibration signals in the time and frequency domains, and calculated the volume, number, and expected volume of segmented objects of the signals in the frequency-energy domain as follows.

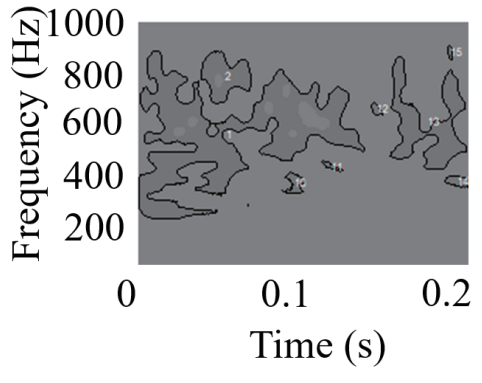

(a)

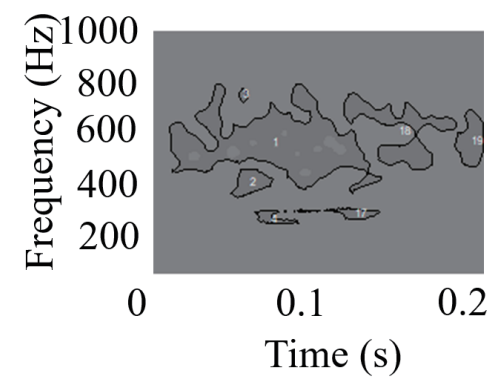

(c)

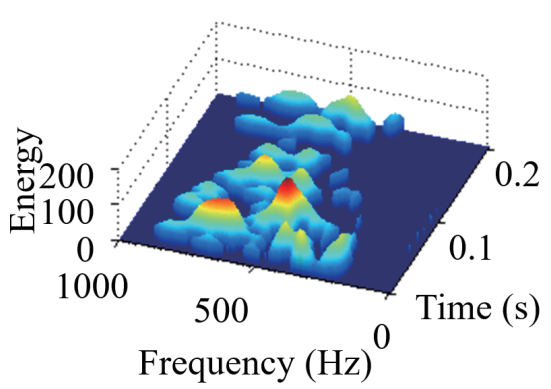

(b)

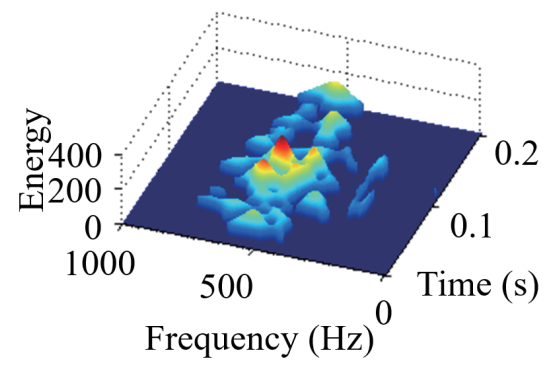

(d)

Fig. 4. (Color online) Results of CWT TFI segmentation. (a) Segmentation results. (b) Characteristic objects. (c) Segmentation results. (d) Characteristic objects. [(a), (b) from test A and (c), (d) from test B]. 


\section{Volume of the signals $\left(V_{m}\right)$}

The volume of a vibration signal is equal to the maximum volume of segmented objects. If the function of the gray area is defined as $f(x, y)$, the maximum volume is calculated as

$$
V_{m}=\iint_{(x, y) \in D_{m}} f(x, y) d x d y,
$$

where $f(x, y)$ represents the energy distribution of the frictional vibration at time $x$ and frequency $y$.

$$
D_{m}=\arg \max _{i}\left\{A\left(D_{i}\right) \mid f(x, y) \geq \tau,(x, y) \in D_{i}\right\}
$$

Equation (5) indicates that the maximum area of the vibration signal in the time-frequency domain reflects the distribution of the energy-dominated vibration at the threshold $\tau$ level. The threshold $\tau$ is satisfied to maximize the variance $\sigma^{2}{ }_{B}$ between the vibration signal and the background noise. Here, the variance is defined as

$$
\sigma_{B}^{2}=w_{0}\left(\mu_{0}-\mu_{r}\right)^{2}+w_{1}\left(\mu_{1}-\mu_{r}\right)^{2}=w_{0} w_{1}\left(\mu_{1}-\mu_{0}\right)^{2},
$$

where $w_{0}, w_{1}, \mu_{0}$, and $\mu_{1}$ represent the normalized area of the segmented object, the normalized area of the background noise, the average energy of the segmented object, and the average energy of the background noise in the time-frequency domain, respectively. The maximum energy variance $\sigma_{B}^{2}$ between the two areas represents the optimal separation of the areas. The corresponding vibration signal is optimally separated from complex signals. According to the above analysis, $V_{m}$ is equal to the energy of the most severe vibration in a certain period, representing the extent of the frictional vibrations.

\section{Number of segmented objects of vibration signals $(N)$}

The number of segmented objects of frictional vibration signals over a period of measurement $(N)$ is counted in $D_{i}$, a region where the energy is concentrated. The areas with the highest energy were found to exhibit the vibration induced by friction. When $N$ increases, the energy becomes more concentrated.

\section{Expected volume of vibration signals $\left(V_{\text {mean }}\right)$}

The expected volume of the vibration signals is defined as

$$
V_{\text {mean }}=\left(\sum_{i=1}^{N} V_{i}\right) / N
$$

where $V_{i}=\iint_{(x, y) \in D_{i}} f(x, y) d x d y$ represents the total energy of the signals $D_{i}$ in an energyconcentrated region and $N$ is the number of objects. Therefore, $V_{\text {mean }}$ represents the average energy generated by each vibration. The expected energy is the average energy and intensity of 
the vibration. The larger the expected energy is, the larger the average energy of the vibration and the more severe the vibration.

\section{Results and Discussion}

\subsection{Test results}

The time-frequency spectrum of frictional vibration signals obtained by the CWT method was plotted, and the vibration features were extracted by the Otsu method to calculate the parameters. Figure 5 shows the relationship between the parameters of the frictional vibration in the two tests.

Figure 5 shows that $V_{m}$ in test A was large at the beginning of the test and decreased to reach its lowest value at $4 \mathrm{~min}$, then fluctuated between 6 and $18 \mathrm{~min}$. In test $\mathrm{B}, V_{m}$ decreased with time and stabilized after $14 \mathrm{~min}$. In general, $V_{m}$ was greater in test B than in test A. $N$ was small for the first $2 \mathrm{~min}$ in test $\mathrm{A}$, then increased rapidly. Its value fluctuated between 4 and $14 \mathrm{~min}$ and fell to a minimum level at $16 \mathrm{~min}$, after which it was relatively stable. The initial $N$ in test B was larger than that in test A, then $N$ rapidly decreased at 4 min and was smaller than that of test A between 4 and 14 min. The value remained relatively stable and then began to increase. $V_{\text {mean }}$ in test A was larger than that in test B at the beginning of the test, and then rapidly decreased to a minimum value at $4 \mathrm{~min}$, after which it stabilized, before increasing rapidly after $14 \mathrm{~min}$. $V_{\text {mean }}$ in test B was larger than that in test A between 4 and $14 \mathrm{~min}$, and smaller than that in test A after 14 min.

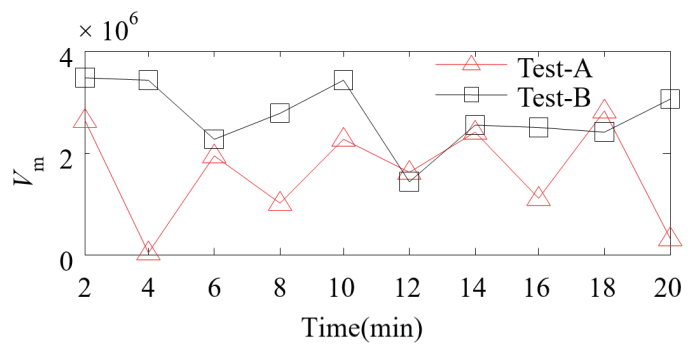

(a)

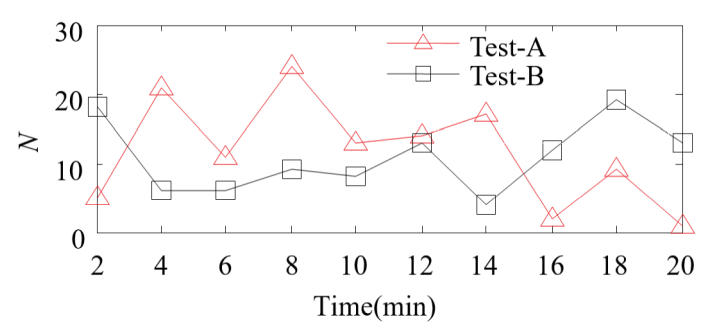

(b)

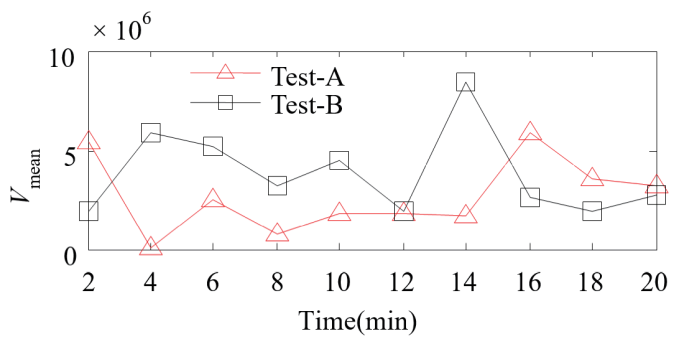

(c)

Fig. 5. (Color online) Parameters as functions of test time. 
The results imply that at the beginning of the tests (before $4 \mathrm{~min}$ ), the frictional vibrations of the two tests were initially severe but decreased with time. The number of vibrations in test A was less than that in test B for the first $4 \mathrm{~min}$, and then increased with time and was larger than that in test B between 4 and 14 min. The vibration was severer in test B and the number of vibrations decreased with time after the beginning of the test. The energy of the vibration in test A was larger than that in test B at the initial phase but became smaller than that in test B between 4 and 14 min.

The variations of the parameters in the two tests were consistent with the extent of wear of the sliding bearing. Because the bearing was boundary-lubricated in test $\mathrm{B}$, the lubrication quality was poor, resulting in boundary friction. At the initial stage of the test, the surface of the friction pair was rough with a large number of micro-convex peaks. The frictional contact of the two surfaces was point-to-point to yield the peaks in the vibration signals. The movements of the friction pair caused the collision of micro-convex peaks. This generated abrasion and scratches, which caused severe vibrations, and the micro-convex peaks were quickly worn away at the beginning of the tests. Then, the intensity of the frictional vibration decreased. The boundary lubrication in test B caused serious adhesion at the initial stage of running-in, generating a large number of abrasive grains. In the middle of the tests, the running-in efficiency in test B was lower than that in test A owing to poor lubrication, producing fewer but larger abrasive grains in test $\mathrm{B}$ than in test A. Large abrasive grains generated a large vibration energy. The efficient lubrication in test A allowed the early wear of the friction pair to be moderate and prevented adhesive wear from occurring. In this case, the worn particles were fine, so the vibration was not severe, and the number of wear grains increased after the initial stage because of the significant grinding action of fine particles in test A.

Figure 6 shows the surface morphology of the bearings observed under a microscope after the tests. In test $\mathrm{B}$ with boundary lubrication, wear marks were prominent and furrows were wide and deep with an uneven distribution. This indicates that the wear particles are large, the wear is severe, and the running-in efficiency is low. However, in test A with immersion lubrication, the friction surface is relatively clean with shallow and narrow scratches, which indicates a large number of small and uniform wear particles. Thus, the wear intensity is

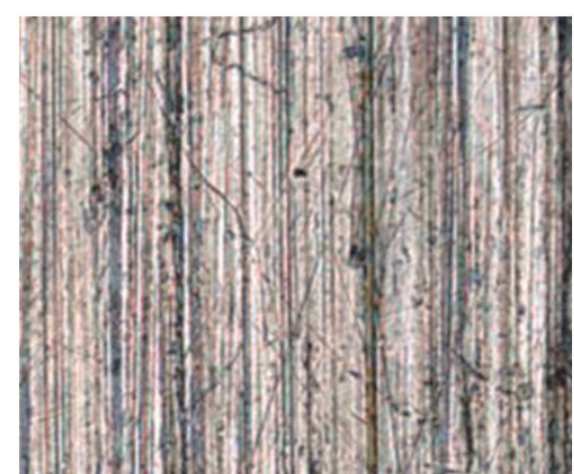

(a)

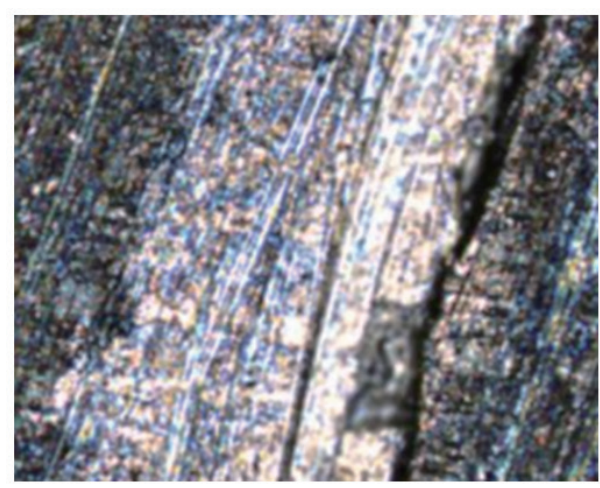

(b)

Fig. 6. (Color online) Surface morphology of bearing alloy samples (100× magnification). (a) Test A. (b) Test B. 
Table 1

Comparison with other methods of analyzing frictional vibration.

\begin{tabular}{|c|c|c|c|c|c|}
\hline Method & $\begin{array}{l}\text { Time domain } \\
\text { information }\end{array}$ & $\begin{array}{c}\text { Frequency domain } \\
\text { information }\end{array}$ & $\begin{array}{c}\text { Spatial } \\
\text { characteristics }\end{array}$ & $\begin{array}{l}\text { Quantitative } \\
\text { analysis }\end{array}$ & $\begin{array}{c}\text { No. of } \\
\text { computations }\end{array}$ \\
\hline Power spectrum analysis ${ }^{(2)}$ & $\mathrm{N}$ & $\mathrm{Y}$ & $\mathrm{N}$ & Y & Small \\
\hline $\operatorname{EMD}^{(3)}$ & Y & Y & $\mathrm{N}$ & $\mathrm{N}$ & Small \\
\hline Fractal analysis $^{(7,10)}$ & Y & Y & $\mathrm{N}$ & $\mathrm{N}$ & Large \\
\hline CWT with image segmentation & Y & Y & Y & Y & Small \\
\hline
\end{tabular}

relatively moderate and the running-in efficiency is higher than that in test B. This result is consistent with the volume, number, and average volume of vibrations. In conclusion, the parameters $V_{m}, N$, and $V_{\text {mean }}$ characterize the frictional vibration signals and the wear of the sliding bearing.

\subsection{Comparison with other methods}

Previous research on frictional vibration signals mainly analyzed the spectral characteristics of vibrations. However, it was difficult to assess the extent of the wear and the health of a friction pair. Table 1 shows a comparison of the information obtained from the CWT with image segmentation and that obtained from other methods. The method proposed in this study provides information in the time and frequency domains. As pointed out earlier in this paper, power spectrum analysis cannot obtain time domain information, and EMD and fractal analysis can only obtain time and frequency domain information. However, quantitative analysis is enabled only by the CWT with image segmentation.

\section{Conclusions}

In this study, we showed that the CWT with image segmentation is an effective method of monitoring the friction and wear behavior of friction pairs because time-frequency images contain abundant information on frictional vibrations. Image segmentation with the CWTextracted information on the frictional vibration of a slide bearing in the time-frequency domain using a simpler algorithm and fewer computations than other methods. The obtained parameters $V_{m}, N$, and $V_{\text {mean }}$ represented the extent, frequency, and energy of frictional vibrations, respectively, and these parameters can be used to characterize the extent of the wear of sliding bearings quantitatively. The proposed method overcomes the disadvantages of conventional methods and does not require the machine under test to be stopped or disassembled. This method is expected to enable the real-time monitoring of the wear of a friction pair. The application of vibration sensors and the analysis of the vibration signals obtained from them will provide a new way to monitor the friction and wear of friction pairs.

\section{Acknowledgments}

This work was supported by the Natural Science Foundation of Liaoning Province of China (20180551145) and the Fund of Fujian Province Key Laboratory of Ship and Ocean Engineering. 


\section{References}

1 P. R. N. Childs: Mechanical Design Engineering Handbook (Elsevier, London, 2019), 2nd ed. Chap. 5. https:// doi.org/10.1016/C2016-0-05252-X

2 D. Sun, G. B. Li, and H. J. Wei: J. Harbin Eng. Univ. 36 (2015) 166. https://doi.org/10.3969/ j.issn.1006-7043.201310041

3 L. Pan: Petro-Chem. Equip. Tech. 40 (2019) 19. http://en.cnki.com.cn/Article_en/CJFDTotal-SHSJ201901005. htm

4 Q. Q. Liu, K. Feng, N. Yang, Y. S. Zhang, and Y. A. Wang: Control. Instrum. Chem. Ind. 45 (2018) 879. http:// www.en.cnki.com.cn/Article_en/CJFDTotal-HGZD201811013.htm

5 G. B. Li, D. L. Guan, and H. J. Wei: Tribology 26 (2006) 461. http://en.cnki.com.cn/Article_en/CJFDTotalMCXX200605014.htm

6 Y. T. Zhang, G. Q. Ren, and G. Z. Li: Trans. CSICE 24 (2006) 459. http://en.cnki.com.cn/Article_en/ CJFDTOTAL-NRJX200605012.htm

7 Q. Zhang, Y. X. Wang, B. Xiong, C. J. Jiang, X. Y. Wang, and C. Zen: Comput. Tech. Geophys. Geochem. Explor. 41 (2019) 181. http://en.cnki.com.cn/Article_en/CJFDTOTAL-WTHT201902006.htm

8 J. Y. Yang, Y. Y. Zhang, and Y. S. Zhu: Mech. Syst. Signal. Process. 21 (2007) 2012. https://doi.org/10.1016/ j.ymssp.2006.10.005

9 H. Zhu and S. R. Ge: Tribololgy 24 (2004) 433. http://en.cnki.com.cn/Article_en/CJFDTotal-MCXX200405011. htm

10 S. Li: Mech. Eng. Autom. 22 (2019) 154. http://en.cnki.com.cn/article_en/cjfdtotal-jxxb703.004.htm

11 C. Y. Hu, J. X. Chen, and X. Q. Yu: Instrum. Tech. 17 (2018) 6. http://en.cnki.com.cn/Article_en/CJFDTOTALYBJI201805002.htm

12 Z. Li, X. C. Chen, and Z. B. Liu: Transact. CSICE 19 (2001) 3. http://en.cnki.com.cn/Article_en/CJFDTotalNRJX200103010.htm

13 Z. Li, X. C. Chen, Z. R. Zhang, and Z. B. Liu: J. Vibra. Meas. Diagn. 22 (2002) 4. http://en.cnki.com.cn/ Article_en/CJFDTotal-ZDCS200204010.htm

14 Z. Li, X. C. Chen, and Z. B. Liu: Trans. CSICE 19 (2001) 3. http://en.cnki.com.cn/Article_en/CJFDTotalNRJX200103010.htm

15 Jinan Liangong Testing Technology: http://www.testermachine.com/testermachinecom/content/?221.html (accessed June 2020).

16 The world material: https://www.chinesesteelgrades.com/45-steel/ (accessed June 2020).

17 C. C. Liu and Z. D. Qiu: J. Circuit. Syst. 5 (2000) 1. http://en.cnki.com.cn/Article_en/CJFDTotalDLYX200001015.htm

18 C. H. Dong, G. Z. Lai, and X. H. Yu: Matlab Image Processing and Application (National Defence Industry Press, Beijing, 2004) pp. 173-175.

\section{About the Authors}

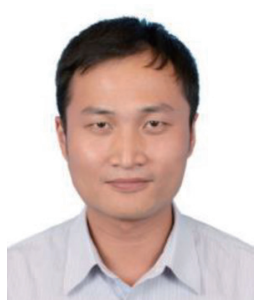

Chaoming Huang received his Ph.D. degree in 2011 from the College of Marine Engineering, Dalian Maritime University (DLMU). He is now a lecturer at DLMU. His major research interests include vibration and noise control, friction and wear control, and fault diagnosis and prediction.

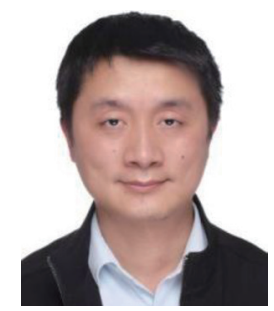

Yongtao Liao started his career at CCCC Shanghai Dredging Equipment Industry Co., Ltd., in 2007 after obtaining a bachelor's degree from the College of Maritime Engineering, DLMU. His major research interests include friction and wear control. 


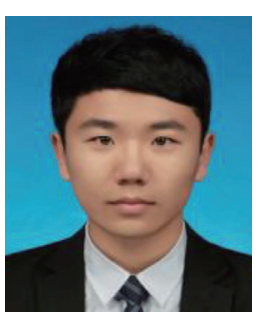

Qingtao Li has been pursuing a bachelor's degree in the College of Marine Engineering, DLMU since 2019. His major research interests include vibration and noise control, and vibration feature extraction.

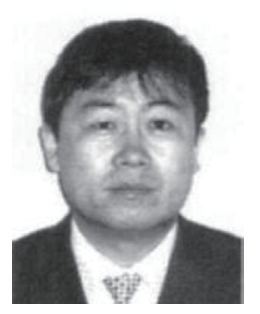

Hongliang Yu received his Ph.D. degree in 2002 from Kobe University of Mercantile Marine, Japan. He is now a professor at Jimei University, Xiamen, China. His major research interests include modern marine engineering management, vibration fault diagnosis, module shipbuilding, and reliability.

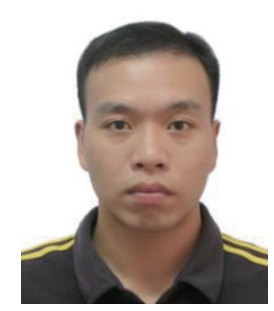

Jianbin Liao is an associate professor at Jimei University. He is currently a Ph.D candidate of DLMU. He received his B.S. and M.S. degrees in marine engineering from Jimei University in 1998 and 2007, respectively. His current research interests include marine engineering and fault diagnosis.

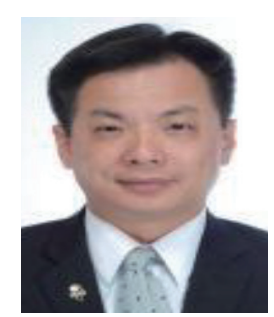

Chin-Cheng Chen has been a professor at Jimei University, China, since 2017. He became a member of IEEE in 2011 and a senior member in 2016. He earned his M.S. and Ph.D. degrees from the Department of Mechatronics Engineering, National Changhua University of Education. His research interests include AIoT technology and RFID applications.

(201761000018@jmu.edu.cn) 